\section{De la vigilancia convencional al monitoreo participativo*}

\author{
From conventional surveillance \\ to participatory monitoring
}

1 Centro de Estudios y Asesoría en Salud (CEAS). Asturias N 2402 y G. de Vera (La Floresta), Quito, Ecuador. jbreilh@ceas.med.ec * Trabajo basado en la ponencia a la Conferencia sobre Salud en el Trabajo y Ambiente: Integrando las Américas - Salvador (Brasil), junio 9 del 2002.

\begin{abstract}
There is a need to transform health monitoring from an instrument of a superficial and passive insight of health, into a tool for critical health assessment, advocacy and collective empowerment; conventional health surveillance, in other words, must be converted to active community driven monitoring. Official information systems are usually describing isolated "disease factors" and associated morbidity outcomes, instead of analyzing the generative processes that make part of the complex determination of health. Subordinated societies have undergone a historical process of assault to human social and health rights; national and international economic exploitation and political-cultural domination have produced an scenario of profound inequity and health deterioration which demand a more penetrating knowledge and information of health scientists and experts. In the face of those needs, this papers exposes the flaws of traditional surveillance and the potentialities of new approaches of health monitoring, based on the new conceptual and practical framework of critical epidemiology
\end{abstract}

Key words Participative health monitoring, Traditional surveillance, Critical epidemiology, Epidemiological profile
Resumen La necesidad detransformar el monitoreo de la salud de un instrumento funcional al sistema dominante de poder en una herramienta del poder colectivo, y de negociación del desarrollo de la salud laboral y el bienestar, implica la urgente transformación de la vieja vigilancia epidemiológica en un monitoreo participativo. D ebe superarse el sistema convencional de información en salud, que observa fundamentalmente los "factores de enfermedad" y sus efectos mórbidos aislados, invisibilizando los procesos de determinación integrales. Este trabajo analiza el proceso de derrota de los derechos sociales y laborales, lo cual sitúa el desafío planteado en un escenario de profundo retroceso social y asalto a los derechos conquistados por los/as trabajadores/as en años de lucha. Con el propósito de esclarecer dicho argumento, al autor comienza contrastando los modelos de vigilancia clásicos, algunas propuestas transicionales, hasta arribar al monitoreo participativo. La aplicación de la categoría "perfil epidemiológico" permite sistematizar los dominios y dimensiones del monitoreo y establecer los sujetos sociales de esa mirada crítica e intercultural del pueblo sobre su salud, mediante la construcción de una matriz de procesos críticos.

Palabras-clave $\mathrm{M}$ onitoreo partici pativo, $\mathrm{Vi}$ gilancia de la salud, Epidemiología crítica, Perfil epidemiológico 


\section{Introducción}

La propuesta que se expone en estas páginas es la de transformar el monitoreo de la salud de un instrumento funcional al poder, hacia un instrumento de poder colectivo para la prevención profunda.

Es definitiva la necesidad de dar un salto desde la vigilancia convencional al monitoreo participativo y, en los momentos actuales, ese paso implica: comprender el escenario histórico de nuestros países y preguntarnos si lo que queremos hacer es vigilar pasivamente los indicadores de la derrota de los derechos laborales o más bien orientar las actividades del monitoreo hacia un proceso participativo de empoderamiento de nuestras colectividades; en segundo lugar, implica reconocer que la vigilancia convencional de "factores de enfermedad" debe dar paso a un monitoreo de determinantes de salud; y finalmente, implica buscar en casos concretos de aplicación el saber y la experiencia necesarios para avanzar.

A lo largo de esta breve exposición desarrollaremos dicha idea y refrescaremos argumentos a favor del monitoreo participativo que fuera propuesto desde hace varios años por algunos núcleos en América Latina, y a la cual dediqué un capítulo entero de mi libro Nuevos conceptos y técnicas de investigación (Breilh, 1997). En los momentos actuales, esas ideas vuelven a cobrar especial urgencia porque enfrentamos unos de los períodos históricos de mayor necesidad de recuperación de la voluntad colectiva para defender una salud seriamente afectada en todo el globo, y aportar a la construcción solidaria de ese "otro mundo posible", que sólo podrá serlo, activando tanto al Norte como al Sur del Río Grande, a lo largo y ancho de las Américas una gestión democrática y contrahegemónica, como alternativa a la gerencia funcionalista que el modelo socioeconómico actual ha impuesto.

\section{Monitoreo de la derrota o de la construcción de otra salud posible (Salud en el trabajo y el monitoreo en el capitalismo actual)}

\section{Trespasos hacia la barbarie el asal to histórico} a los derechos humanos y laborales

Hemos insistido en que nadie duda en la actualidad que en las Ilamadas "democracias" del Norte y del Sur el fundamentalismo liberal nos ha arrojado hacia una absurda carrera, en la que los países compiten, en realidad, por cual va a llegar primero a ser el peor en términos humanos para lograr la mayor tasa de explotación, tanto del ser humano, como de la naturaleza.

La sociedad capitalista hace varias décadas dejó de ser el escenario de esa clásica explotación de la fuerza de trabajo y extracción de una tasa de plusvalía, mediada por condiciones básicas de seguridad laboral y bienestar social; ahora se trata de un sistema que agudizó los términos de la explotación y la dominación social, al punto que ha degradado la subsistencia a lo mínimo y ha creado la imposibilidad estructural para una reproducción social y ecológica sustentable.

En otros textos hemos explicado cómo a nuestro entender se llegó a este retroceso profundo de los derechos humanos, sociales y específicamente laborales que ahora confrontamos (Breilh, 2003a e b).

En un primer momento de la sociedad existió un sujeto comunitario. Eran épocas en que los sujetos sociales operaban en función de valores de uso, que satisfacían necesidades establecidas por conveniencia colectiva. En esos tiempos ni la división sexual del trabajo, ni las diferencias de género, ni los contrastes étnicos provocaron desigualdades importantes; no porque se hayan tratado de sociedades ideales, sino porque el grado de desarrollo exigía equidad para la supervivencia.

La conquista y la instauración de la sociedad colonial fracturó el sujeto comunitario, y los atomizó en sujetos privados, guiados por el afán de atesoramiento mercantil. Así fueron rotos el derecho igualitario y los principios de equidad, y junto con ellos se derrotó la concepción armónica del manejo de los bienes de la Naturaleza. La expropiación del oro y de la tierra, y la explotación feudal de la fuerza de trabajo fueron la base de la economía del mercantilismo colonial, provocándose la primera derrota de los derechos humanos y de la necesidad como eje de la construcción social. En la entraña de esa primera etapa de la inequidad humana, se incubó una concentración de poder que reprodujo una triple jerarquización de la calidad de vida y del trabajo: la de pertenencia a una clase; la de pertenencia a una etnia; y la de pertenencia a un género. Triple inequidad social que para reproducirse requería de un Estado uninacional y la uniculturalidad como forma de cultura oficial. 
Ya entrada la época de nuestras repúblicas, sucedería la segunda gran derrota de los derechos humanos y de la necesidad como principio de definición social, cuando las mujeres y hombres pobres perdieron el derecho a la propiedad de los bienes fundamentales de la sociedad industrial. Aunque el Ilamado "sujeto obrero" logró - sobretodo en las sociedades industrializadas - mantener con su lucha la vigencia de al gunos derechos laborales, sociales y culturales básicos, aquel nuevo orden económico no permitió, con todo, que la política de pleno empleo se extendiese a las naciones periféricas y se difundiesen más equitativamente los be neficios. Bajo la férula de relaciones neocolonialistas, esas naciones se tornaron exportadoras de productos primarios y se vieron impedidas de asumir a fondo la industrialización, con lo cual sólo un modesto porcentaje de su población conquistó los derechos humanos y sociales del antes mencionado Pacto de la Segunda Posguerra (Frei Betto, 2002). En esas condiciones se consolidó la subordinación no sólo de los trabajadores como clase, sino de los sujetos étnicos - indígena y afroamericano - y del sujeto femenino, mediante la instauración de un aparato educativo y políticas culturales eurocéntricas y androcéntricas.

Finalmente, desde mediados de los años 80 se acentuó el carácter concentrador y monopólico de la economía y se abrió el nuevo período de la mercantilización global. La maquinaria neoliberal y sus mecanismos de recomposición productiva han provocado un cataclismo económico social y laboral, y para sostenerse renuevan la estrategia del terrorismo oficial; sólo que ahora la opresión no se presenta en la forma directa de las dictaduras, sino que se ejerce a través de la administración del miedo y la inseguridad como forma de gobierno. Comenzó así la tercera y más profunda derrota de los dere chos humanos y una polarización sin atenuantes de la sociedad. Es un modelo social perverso, porque aunque teóricamente se esgrime como una salida a los errores del keynesianismo y como un proyecto para colocar la riqueza acumulada por las empresas al servicio de la gente, lo que en verdad se ha montado es una maquinaria de demolición de los derechos, un mecanismo institucional y jurídico para imponer políticas de minimización de la canasta familiar a niveles de hambre e institucional izar la apropiación fraudulenta de los recursos estratégicos, de los fondos de ahorro y jubilaciones de los ciudadanos. Al haberse constituido aho- ra más que nunca la vida humana alrededor del afán productivo, y la producción al rededor del sediento cálculo de intereses de los monopolios privados, las actividades productivas menosprecian los impactos de tal actividad sobre la vida (humanidad y naturaleza), de ese modo el crecimiento económico está impedido de ser una vía de desarrollo humano. Es tan serio el retroceso de las políticas laborales y sociales orquestado por el Estado neoliberal que Galeano se pregunta ahora con incisivo humor, si los derechos de los trabajadores no serán un "tema para arqueólogos".

Y no siempre la dominación se impone con violencia pues ahora buena parte de la fuerza laboral acepta esas reglas del juego. De ahí surge una pregunta clave: ¿Cómo entender la paradoja de una masa que se ahoga en la necesidad y la pobreza sin tener claridad sobre el carácter explotador del sistema en su conjunto? Existen dos tipos principales de explicación, para autores como Negri \& Hardt (2000) el cambio esencial que define la época y sus comportamientos sería el paso de una fase de dominio social mediante aparatos que producen y regulan costumbres, hábitos y prácticas, hacia una sociedad en la cual los mecanismos de tal dominio se ejercen a través de los cuerpos y las mentes de los sujetos individuales; algo así como una extrapolación de la "subsunción real", originalmente descubierta por $\mathrm{M}$ arx en el control de los trabajadores por los mecanismos internos del proceso productivo, hacia la cultura y los comportamientos en general; un poder ...ejercido por medio de máquinas que, directamente, organizan las mentes (en sistemas de comunicaciones, redes de información, etc.) y los cuerpos (en sistemas de bienestar, actividades monitoreadas, etc.) ... (N egri \& Hardt, 2000). Lo único que existiría ahora es una "multitud" diversa y dispersa en el globo, que golpearía al Imperio de manera desarticulada. De acuerdo con esa lectura, los(as) trabajadores(as) se enfrentarían a un poder que está en todas partes y a la vez en ninguna, no tendrían frente a sí de manera tangible y concreta ni a una empresa, ni a un Estado, como tampoco formarían un sujeto social de clase y deberían más bien disolverse en la lucha de la multitud.

Pero otros autores han desnudado los monumentales errores de ese tipo de análisis y han rescatado una visión verdaderamente contrahegemónica (Borón, 2002). Desde esta perspectiva la supuesta desaparición de los estados nacionales (M észáros, 2001) y de los de nexos 
que las empresas transnacionales conservan con éstos es sólo una ilusión pues, lejos de perder toda territorialidad y nexo con Estados concretos ...las transnacionales han constituido una verdadera dictadura mundial, con un mando centralizado, dependiendo de sus propios Estados. Es el caso, por ejemplo, de las cien trasnacionales más importantes de la lista de la revista "Fortune", publicación que encontró que todas ellas se habían beneficiado de intervenciones específicas de los Estados nacionales donde tienen su base, mediante subsidios que provienen del contribuyente fiscal y del desangre del aparato productivo público en ben eficio de las corporaciones (Corbière, 2002).

Los centros de poder monopólico no son virtuales y difusos; esa es sólo la apariencia que ofrece el carácter virtual de sus redes de comunicación instantáneas; ese es el nuevo fetichismo de la comunicación virtual de las empresas. Aquellos centros de poder tienen una organicidad y una correspondencia territorial; están enclavados en los aparatos de Estados concretos; se respaldan en ejércitos regulares o mercenarios que tienen fuentes de financiamiento $y$ centros de comando insertos especialmente en los servicios de inteligencia y los ejércitos del principal Estado imperialista - los Estados Unidos; las fuerzas policiales conforman aparatos represivos que subyugan toda movilización en defensa del trabajo o el ecosistema; y finalmente, la hegemonía y el control cultural también se ejercen desde maquinarias, medios de comunicación y aparatos culturales, cuyos centros de conducción radican en dicho Estado.

En un escenario de esas características es urgente e inevitable activar un pensamiento crítico acerca de los problemas de la salud en el trabajo, y para eso es indispensable recuperar una ideología emancipadora. La perspectiva desde la cual nosotros trabajamos es la del neohumanismo popular, y es con base de ese pensamiento que empujamos un paradigma distinto de las ciencias de la salud y de las técnicas como el monitoreo.

No tiene sentido seguir con esa inocua y reduccionista vigilancia epidemiológica que se brinda ingenuamente para vigilar, sin cuestionar, los efectos de la derrota coyuntural de los trabajadores en el mundo. Tenemos que poner en marcha todo el arsenal científico y tecnológico forjado por muchas generaciones de científicos y profesionales de la salud colectiva, activar todos los núcleos del saber, todas las bases de datos, para desentrañar, junto con nuestro pueblo, intercultural mente, la manera de lograr la humanización de los modos de trabajo y consumo, de nuestras relaciones con la madre naturaleza; para potenciar los valores culturales que resalten la dignidad, valoricen la cultura propia y permitan el empoderamiento de la gente, con su plena participación en un sistema de salud conducido por las colectividades.

La salud colectiva reúne un poderoso arsenal de instrumentos para la comprensión de los procesos determinantes de la salud de todos los grupos sociales. Nuestro aporte desde la Epidemiología Crítica Latinoamericana ha sido el desarrollar un sistema de categorías y una propuesta metodológica que permitan entender todas esas dimensiones de la determinación de la salud inscritas en los modos de vida típicos de cada grupo. Hemos podido demostrar que el perfil epidemiológico de un grupo es en definitiva el movimiento de la contradicción entre los procesos protectores y destructivos que operan en su modo de vida característico, y que a la vez, ponen límites posibles a los estilos de vida individuales de las personas que lo integran. (Se anexa la matriz de procesos críticos que hemos elaborado para los(as) trabajadores (as) de la floricultura para que se comprenda la utilidad del perfil epidemiológico en el estudio y monitoreo de la salud de dicho grupo).

En la medida en que un sistema social como el presente instituye la proliferación y profundización de formas de inequidad y el consiguiente deterioro de los derechos humanos en esos modos de vida de los trabajadores, entonces se instituyen mecanismos de deterioro de su salud, que afectan sistemáticamente los patrones fisiológicos y las normas de reacción genética de los organismos y funciones fisiológicas de sus integrantes, apareciendo entonces patrones de morbilidad y un sobre-envejecimiento en esa población. Por el contrario, cuando se abren posibilidades de disfrute de una equidad que permite la satisfacción de los derechos, con lo cual se potencian los soportes personales y familiares, así como las defensas y posibilidades fisiológicas, entonces se eleva la calidad de vida fisiológica y genética, y aparecen patrones de salud mental y física, así como mejores índices de sobrevivencia. La vida y la salud de las(os) trabajadoras(as) dependen de ese movimiento entre los procesos que nos protegen y los que nos deterioran, y el desarrollo de todos ellos depende de la capacidad de disfrute de los derechos humanos. 
En esta concepción que estamos defendiendo no hay cabida para una noción ingenua o puramente natural de los procesos biológicos, pues éstos no son puramente biológicos sino socio-biológicos y, en última instancia, expresan en nuestros cuerpos lo que sucede en el orden social. No hay cabida, tampoco, para la noción de la salud como algo esencialmente personal y que depende primordialmente de medidas curativas y de todo este conjunto de procesos del perfil epidemiológico se desprenden las dimensiones y contenido que deben abarcar el monitoreo de la salud de los trabajadores.

Por lo dicho, no podemos encerrarnos en el simple perfeccionamiento técnico de la llamada vigilancia, sino ir hacia una construcción distinta de un verdadero sistema de inteligencia social, construido participativamente sobre la salud y sus determinantes.

Un desarrollo detallado de nuestra propuesta - para cuyo análisis detenido remitimos al lector a varios escritos anteriores (Breilh, 1997; 1999a e b; 2002) - rebasa los límites de esta pre sentación sucinta, pero caben aquí destacarse tres puntos que consideramos deben resolverse en el camino de implementar el nuevo modelo: a) contrastar con claridad el modelo clásico de vigilancia con el monitoreo; b) integrar el monitoreo en un paradigma renovado de gestión en salud; y c) incorporar en el modelo de conocimiento la construcción intercultural como ve hículo de una participación colectiva.

\section{Vigilancia de factores de enfermedad versus monitoreo de determinación de la salud}

La distinción se establece primero por la forma de concebir el objeto salud. M ientras la vigilancia convencional concibe al objeto salud como un proceso esencialmente individual (casos), que se asume por el efecto negativo (enfermedad); en cambio el monitoreo comprendelos procesos críticos de una colectividad (determinantes protectores y destructivos de la salud), que se dan en modos de vida grupales y dentro de estos las expresiones en los estilos de vida familiares personales y las condiciones de los organismos y psiquismo individuales (Figura 1).

En segundo lugar, mientras la vigilancia convencional interpreta la génesis de las enfermedades desde una visión empírica reduccionista, enmarcada en el paradigma positivista, que sólo le permite llegar a la prevención etio- lógica; el fundamento del monitoreo es la epidemiología crítica y la construcción intercultural y planeación estratégica de los procesos críticos del monitoreo.

En tercer lugar desde el punto de vista de la gestión, o de las implicaciones para la acción, la vigilancia clásica se concibe verticalmente desde el aparato del Estado, implicando una posición pasiva de los(as) trabajadores(as) y en el mejor de los casos una colaboración marginal por parte de la gente; este enfoque se realiza bajo una lógica centralizada, que conlleva además en la práctica una mayor ineficiencia y costos, cobertura limitada, un flujo apenas ascendente de la información y una construcción sólo académica del conocimiento. Mientras que en el modelo contrahegemónico, el monitoreo es parte de una gestión cuyo eje es la planeación estratégica y el control colectivo de la gestión.

\section{El monitoreo como elemento de un paradigma renovado de gestión}

\section{La gestión colectiva: definición de sujetos, necesidad, calidad y control}

Con la finalidad de remontar los estrechos límites de una gestión tecnocrática, hemos pro-

\section{Figura 1}

Diagrama de perfil epidemiológico.

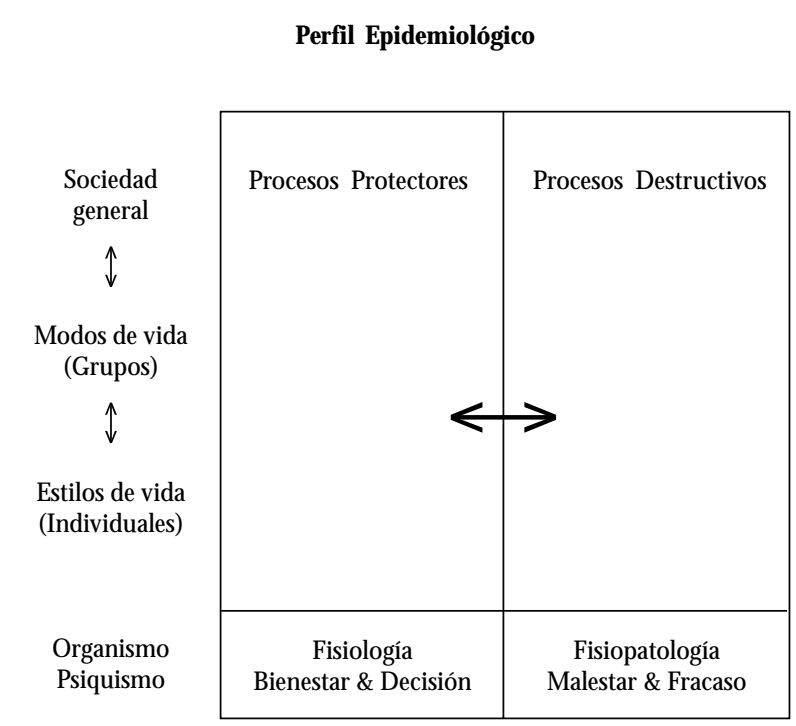


puesto debatir mecanismos para enlazar la gestión del conocimiento institucional con la gestión colectiva del saber. Dicho enlace tiene fundamentos teóricos e implicaciones prácticas para la gestión en su conjunto.

Los avances de las ciencias administrativas aplicadas al desarrollo y las nuevas teorías sobre la gestión - y la gerencia como una de sus herramientas - han determinado la creciente necesidad de discutir las articulaciones entre el esfuerzo social y la gerencia. $Y$ aquí vuelve a aparecer la necesidad de un debate esclarecedor.

$\mathrm{H}$ emos sostenido en varias oportunidades que el monitoreo participativo constituye, junto a la planeación estratégica y al control social, un pilar de una gestión innovadora de la salud.

En el campo del desarrollo social nos vemos avocados a abrir la gestión a la colectividad y enlazar no sólo conceptualmente sino técnicamente los procesos de la gestión del conocimiento.

En esa misma dirección, vale recordar los criterios de validez de cualquier discurso del conocimiento, denominados por $\mathrm{H}$ abermas como "niveles de validación intersubjetiva": a) la capacidad del discurso para favorecer la implementación de condiciones favorables para el éxito de proyectos sociales definidos y acciones legítimas a los mismos (validez normativa); b) la proposición de enunciados que puedan ser compartidos por distintos sujetos (validez proposicional); y c) su capacidad para establecer una comunicación efectiva y expresar

\section{Figura 2}

Secuencia del disenõ participativo.

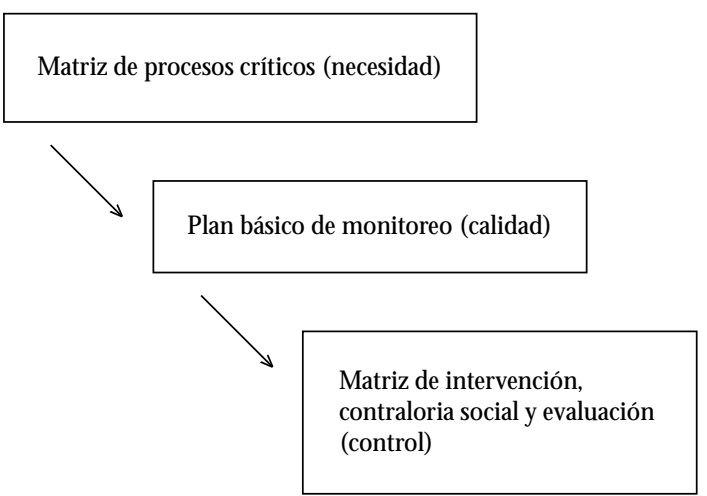

las distintas perspectivas o sujetos que entran en interacción (autenticidad expresiva) (H abermas, 1998; Ayres, 1997).

Una gestión en la que converjan un pensamiento crítico sobre la gestión y una población organizada e informada adquieren un poder decisivo para transformar los problemas y para mantener un proceso de avance del conocimiento y del saber. Y la planeación del saber juega un papel clave tanto en la construcción de dicho pensamiento crítico, cuanto en el impulso de una participación informada.

La gestión colectiva puede canalizarse básicamente a través de tres mecanismos: la planeación estratégica; el monitoreo estratégico; y el control social sobre la gestión (Figura 2).

La planeación estratégica es el proceso de construcción de un proyecto colectivo, con autarquía y claridad de objetivos estratégicos, en un escenario de inequidad social y bajo un estructura de poder (de clase, género y etno-cultural). Es un movimiento de gestión del saber y de intervención organizada de dicho colectivo en busca de metas sociales democráticamente definidas. Ese movimiento implica varios momentos que no se realizan de manera lineal o mecánica, pero cuyo desarrollo va gradándose según las demandas de intervención y gestión del saber: 1) momento de construcción de una matriz de procesos críticos (en la que se formulan los elementos del perfil epidemiológico sobre los que se enfoca el conocimiento y la acción; relacionados a grupos clave y situados en un territorio social y ecosistema; 2) un momento explicativo en el quese establecen las relaciones determinantes y procesos generativos de aquellos procesos críticos; 3) un momento político en que se esclarecen los lineamientos y agenda de la acción, y se analizan las fuerzas involucradas (favorables y antagónicas); 4) un momento de análisis propiamente estratégico en el que asumen las prioridades y las líneas de acción; 5) un momento operativo.

El monitoreo participativo es la mirada permanente de la colectividad organizada sobre los procesos de los que dependen su bienestar, su funcionamiento democrático y la reproducción de sus conquistas materiales, culturales y humanas. El monitoreo se realiza sobre indicadores o procesos trazadores de cada uno de los dominios, dimensiones, determinantes estructurales, procesos generativos y procesos específicos de salud de los grupos involucrados (ver matriz de procesos de intoxicación). 
El control social es participación concreta del pueblo organizado en la evaluación y ajuste de los procesos en su conjunto, y en los escenarios de toma de decisiones, sobretodo en aquellos en que se decide sobre la asignación de recurso y presupuestos. Dicha posibilidad se ejerce por medio de diversos canales y tanto en espacios locales, como regionales, cuanto nacionales. Existen experiencias tanto en el país, como en otras sociedades latinoamericanas Brasil, Uruguay, Argentina, Venezuela y otras que demuestran que la participación del pueblo no sólo contribuye a frenar la corrupción estructurada que campea, ni sólo insume eficiencia en la gestión, sino que aporta en una verdadera recomposición de la política.

\section{La gestión colectiva: ¿Q uiénes y cómo definen la necesidad, la calidad y el control de los procesos en un espacio laboral?}

Son tres los parámetros básicos que definen el carácter de una gestión: la necesidad; la calidad y el control. (Figura 3)

En la planificación normativa y en las entidades donde se impone una gestión vertical, son los representantes del poder los que terminan definiendo en última instancia lo que se necesita, la calidad de las acciones que se tolera y las formas de control de la gestión y su financiamiento. Bajo esa perspectiva el monitoreo solo es una vigilancia de los efectos de la acción del aparato institucional y de algunas de las funciones que realiza. En cambio bajo una gestión participativa y la planeación estratégica, el monitoreo es la mirada de la colectividad y el seguimiento de la construcción del proyecto colectivo y de los obstáculos que enfrenta. En el marco de este monitoreo no puede enfocarse solo la morbi-mortalidad sino el conjunto de los procesos de la matriz: procesos estructurales, procesos generativos y, a la luz de aquellos procesos específicos de la salud (no sólo de la enfermedad). Ver ejemplo de la matriz de intoxicación en la agroindustria florícola.

\section{La interculturalidad en la construcción de la "inteligencia popular"}

En otro trabajo expusimos ampliamente la importancia de la intersubejtividad en el desarrollo del modelo praxiológico que proponemos, en el marco del realismo dialéctico. Y como lo dijéramos entonces, nuestro análisis epistemológico implica un doble movimiento. Por un lado, la búsqueda consciente de articulación de la epidemiología crítica a un modelo de desarrollo emancipador; es decir, la procura de una configuración epidemiológica instrumental a dicho modelo - tarea que conlleva la construcción de una praxis orientada hacia la equidad, contra el poder hegemónico y por la reestructuración y fortalecimiento de una construcción colectiva del saber. Una forma de ejercer la intelectualidad orgánica - en el sentido gramsciano - , que se oponga a la descomunitarización e instrumentalización funcional de dicho saber. En ese sentido, tiene que ser un movimiento doblemente concatenado, tanto con la totalidad social, como con la localidad (Breilh, 2003b).

La interculturalidad es una relación estratégica o dialógica entre culturas (dependiendo de

\section{Figura 3}

Parámetros básicos.

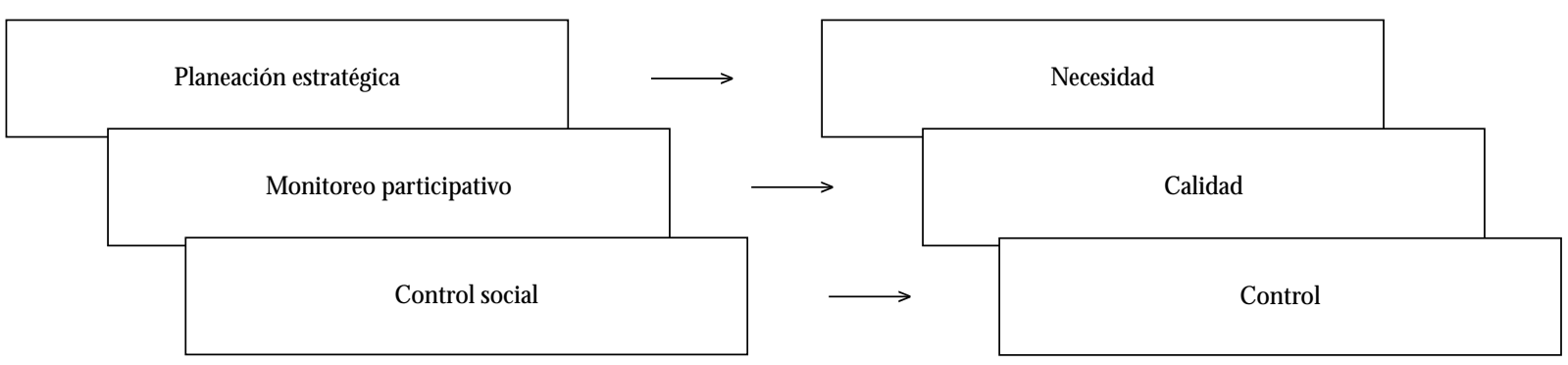


las relaciones sociales entre los sujetos involucrados), con objeto y propuesta global integrados, para construir contraconstruir/reconstruir en el conocimiento de los procesos de la realidad. La interculturalidad presupone equidad de derechos; reconocimiento y respeto por la cultura de los(as) otros(as); así como reconocimiento de características social es y metas estratégicas socialmente compaginadas.

Sobre la base de un trabajo intercultural se puede construir un multiculturalismo crítico y democrático, que involucra un programa, políticas y movimiento nacido del reconocimiento de la diversidad y derechos culturales. Es la integración de varias culturas, por medio del análisis intercultural, y en medio de una visión cultural solidaria, para la construcción de un proyecto común de democratización y equidad.

La construcción intercultural del saber y de un sistema de monitoreo no se reduce a que el sujeto académico o el experto técnico capten y replanteen las evidencias constatadas y construidas desde otras culturas y otras formas de producción de conocimiento, es más bien un desafío para perfeccionar un proceso intercultural que permita, como diría Walsh (2001), la traducción recíproca de conocimientos, la construcción conjunta del objeto de transformación y del marco interpretativo, la aparición de nuevas formas de análisis y la aparición de conceptos renovados. Solo así podremos superar el eurocentrismo eiluminismo que han inspirado la tarea de los "expertos occidentales" y podremos construir un saber evaluador de la realidad en salud.

La pregunta correcta no se refiere a si podemos combinar en la producción de conocimiento, la ciencia y el sentido común, sino interrogarnos sobre la posibilidad de integrar el conocimiento académico con los otros sistemas de saber complejos y altamente sistematizados que resumen 0 acumulan una sabiduría fundamental, tanto en la dimensión filosófica como más factual e instrumental. De lo que se trata es triangular el poder de observación y explicación de esas fuentes en los proyectos de acción concretos que llevamos adelante para impulsar el desarrollo humano.

El movimiento del conocimiento evaluador de la calidad de la salud en una colectividad entonces tendría varios momentos y espacios de realización, e implicaría el interjuego de diversas lógicas. La producción del conocimiento de monitoreo a su vez involucra tanto el momen- to de generación del conocimiento (es el movimiento de génesis de productos del conocimiento, que incluye la elaboración de conceptos y la realización de un método, mediante la formulación de descripciones y explicaciones en un escenario práctico), cuanto el de reproducción del conocimiento (Figura 4).

Pero además de la producción de conocimiento la planeación del saber incluye la gestión del conocimiento, que es la forma conciente y planificada de uso y administración del conocimiento de monitoreo producido. El monitoreo no es otra cosa que una gestión de saber estratégica y enfocada en la evaluación de calidad (Figura 5).

En el proyecto EcoSalud que lleva adelante el CEAS, se ha iniciado el proceso de construcción intercultural integrando saberes. Desde el lado comunitario mediante el aporte del conocimiento ecológico y la elaboración de mapas comunitarios; bioensayos simplificados; y el saber epidemiológico popular, registrado también mediante mapas de procesos destructivos reconocidos por la colectividad. El conocimiento académico aportará los elementos que se describen más abajo al hablar de la interdisciplinaridad.

Gramsci puso de relieve hace muchos años la importancia de la relación entre la colectividad organizada y sus intelectuales, y habló de que un proceso emancipador arranca sólo cuando los intelectuales se hacen pueblo y el pueblo se encarna en un pensamiento emancipador de su intelectualidad. Una relación que debemos sopesarla con atención pues cualquier relación entre un grupo de intelectuales y una población constituye una relación emancipadora. Si el vínculo es entre una colectividad carente de una actitud y pensamiento emancipador y un grupo de "expertos" con una mentalidad funcionalista, lo mejor que puede acontecer es un proceso reformista o una activación intrascendente. Mientras que si el vínculo se da entre una colectividad con un pensamiento emancipador y una organización solidaria, y unos expertos con un pensamiento y actividad científica contrahegemónica, entonces si despegan acciones con un profundo impacto transformador.

Para dar vida a esa relación planteada el CEAS aplica este modelo al monitoreo participativo de la floricultura en una de las más importantes regiones de producción de flores cortadas del mundo. Sistema cuya explicación re basaría los límites de este exposición y cuyos li- 


\section{Figura 4}

La construcción participativa del saber.

\section{Planeación del saber}

(A) Producción

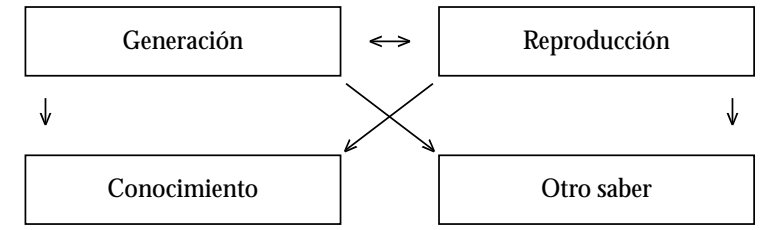

Académico

("Occidental")

Ciencia de lo Concreto

Sistemas M íticos
(B) Gestión

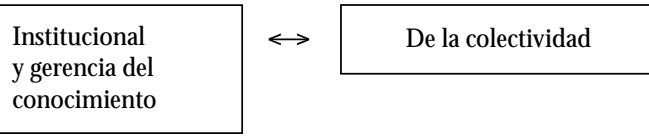

neamientos fueron expuestos audiovisualmente. Eso es lo que se está tratando de desarrollar mediante los sistemas organizativos y de gestión que hemos explicado.

Nuestros esfuerzos representarán el lado humano de la globalización que tendrá que seguir alimentándose del talento, la experiencia y la tenacidad del trabajo que hacemos en Norte, Centro y Sur América gentes que junto a nuestros pueblos cultivamos esa "otra salud posible" que refleja la victoria de las fuerzas de la vida sobre las fuerzas de la muerte.

\section{La interdisciplinaridad crítica}

M as también en el lado académico la complejidad de nuestros objetos de conocimiento requieren la mayor parte de veces una coordinación interdisciplinaria.

La interdisciplinaridad es una relación simétrica, activa de colaboración sistemática entre disciplinas al rededor de una construcción/ deconstrucción/contraconstrucción de objeto de estudio.
Para abreviar la explicación de construcción interdisciplinaria en el proyecto EcoSalud se exponea continuación un cuadro donde pue den reconocerse las contribuciones especializadas (Cuadro 1).

Es decir la mirada sobre lo complejo requiere de la contribución de las distintas miradas académicas que dejamos expuestas, y de éstas con las miradas surgidas de la sabiduría de las y experiencia de las colectividades. Claro está, y como dijéramos antes, la coherencia del metadiscurso crítico que así puede construirse debe ser el sentido emancipador. En otras palabras, desde una perspectiva crítica tendríamos que integrar los elementos críticos emancipadores de todas las miradas y así conformar una metacrítica del sistema social imperante y de la determinación de la salud.

En el caso del ejemplo que hemos utilizado de la floricultura, los ámbitos del problema rebasan las fronteras de una región y se abren al contexto internacional, motivo por el cual se abre la perspectiva del monitoreo al nivel internacional (Figura 6). 
Figura 5

Elementos para una construcción democrática de la gerencia en salud.

\section{Planeación del saber}

Gestion del conocimiento

(Espacio institucional)

- Generación colectiva (general o por grupos homogéneos - comunidades de práctica)/individual de un modelo de conocimiento flexible e interactivo; innovador; formal/informal

- Estrategia de vigorización de la producción y uso de conocimientos (ambiente del conocimiento)

- Red de acceso/

Uso de conocimientos

- Representación (bases datos

- cualitativos/cuantitativos)

- Inserción (en productos/servicios)

- Transferencia de conocimientos/ prácticas

-Uso en decisiones

- Crescimiento cultural e incentivos

- Sistema de evaluación

(impacto y funcionalidad)
Gestión colectiva

(Espacio de la colectividad)

-Determinantes económicos,

culturales, y políticos

(Análisis contextual)

- Planeación estratégica

- Monitoreo

- Control social
Finalmente, para ilustrar sobre la necesidad de que la observación epidemiológica aborde todos los dominios y dimensiones que hacen parte de la salud como objeto multidimensional y contradictorio exponemos la matriz de procesos críticos (Cuadro 2) que hemos empleado para organizar nuestro trabajo de investigación e intervención.

En definitiva, creemos que podríamos aplicar a la discusión sobre el monitoreo lo que dijéramos para la epidemiología en general: ...la Epidemiología crítica va en busca de una nueva visión de la ciencia, de lo que es el conocimien- to duro y de lo que es una intervención eficaz; no quiere ser apenas un nuevo arreglo de viejas ideas y fórmulas funcionalistas, vestidas con el ropaje seductor de una tecnología de punta (Breilh, 2003).

Nuestros esfuerzos representarán el lado humano de la globalización que tendrá que seguir alimentándose del talento, la experiencia y la tenacidad del trabajo que hacemos en Norte, Centro y Sur América gentes que junto a nuestros pueblos cultivamos esa "otra salud posible" que refleja la victoria de las fuerzas de la vida sobre las fuerzas de la muerte. 


\section{Cuadro 1}

Hacia una interdisciplinaridad crítica.

\begin{tabular}{lll}
\hline Dominio & Proceso Crítico & Disciplina
\end{tabular}

Lógica estructural determinante:

- Productiva

- Consumo

- Estado

- Condiciones geoecológicas

Particular (obreros flores)

- Modos de vida y sus cambios

- Impactos culturales

- Formas organizativas

- Relaciones ecológicas
Recomposición agroindustrial Sistema de comercio internacional Desregulación Pérdida de biodiversidad
Economía agrícola

Economía

Sociología

Ecología
Sistema productivo y tipología tecnológica

Transformación de cultura agrícola tradicional a cultural agro empresarial.

Modificación de patrones

Cambio de organización comunal

Dinámica de los plaguicidas en el ecosistema Perfiles de exposición y manejo ecológico
Economía agrícola, sociología

Antropología

\section{Sociología, antropología}

Ecología

Antropología, ecología, epidemiología

Antropología,epidemiología Epidemiología, laboratorio clínico

\section{Figura 6}

Elementos para el monitoreo en ámbito internacional.

Floricultura: campos para investigación/monitoreo/certificación

\section{Práctica empresarial}

Naturaleza y lógica: Concentradora

(monopólica)

vs.

Redistributiva
Procesos críticos de exposición

Estudio de los impactos humanos

(genotípicos y fenotípicos) 


\section{Cuadro 2}

Matriz de procesos críticos del perfil de intoxicación.

\begin{tabular}{|c|c|c|c|}
\hline \multirow[t]{2}{*}{ Dominios } & \multirow[t]{2}{*}{ Dimensiones } & \multicolumn{2}{|c|}{ Determinantes estructurales } \\
\hline & & Destructivos & Protectores \\
\hline \multirow[t]{9}{*}{ GLOBAL } & Lógicadeterminante & & \\
\hline & - Productiva & • Recomposición & $\begin{array}{l}\text { - Producción justay } \\
\text { ecológica }\end{array}$ \\
\hline & & • Flexibilización & - Maneo ecológico integrado \\
\hline & & $\begin{array}{l}\text { - Modelo de producción } \\
\text { y tecnológico de } \\
\text { la revolución verde }\end{array}$ & \\
\hline & - Consumo & • “Flor perfecta” & $\begin{array}{l}\text { - Consumo deflor justay } \\
\text { ecológica }\end{array}$ \\
\hline & - Estado & - Desregulación & $\begin{array}{l}\text { - Códigos protectores, } \\
\text { nacionales einternacionales }\end{array}$ \\
\hline & Condiciones geoecológicas & $\begin{array}{l}\text { - Distribución antitécnica } \\
\text { deuso desuelos }\end{array}$ & $\begin{array}{l}\text { - Asignación planificada } \\
\text { deuso desuelos }\end{array}$ \\
\hline & & - Deterioro desuelos & $\begin{array}{l}\text { - Protección composición } \\
\text { desuelos }\end{array}$ \\
\hline & & - Pérdida debiodiversidad & - Protección biodiversidad \\
\hline
\end{tabular}

Procesosgenerativos

Procesosesp. desalud

Destructivos Protectores


Cuadro2 (continuación)

Dominio

Dimensones

Determinantes estructurales

Procesosgenerativos

Procesosesp. desalud

Destructivos

Protectores

Destructivos

Protectores

\section{PARTICULAR}

\section{Modosdevida}

- Patrones detrabajo

- Patrones deconsumo

- Cosmovisión y perfil desubjetividad

- Formas organizativas

- Relaciones ecológicas particulares
- Distribución de procesos

nocivos por áreas:

Modelo productivo

Subsunción o falta control

del trabajador

Alta exigencia cíclicay

no periodos adecuados

dereposo;

Extremos y fluctuación

térmica;

Deshidratación;

Movimientos repetitivos:

Forzamientos posturales:

Transporte pesos;

Inadecuación ergonómica

depuestos;

Ruido / vibraciones;

Exposición / imposición

a plaguicidas

- Limitación y deformación alimentaria

- Conformismo; pérdida deidentidad

\section{- Alineación,}

no organización

- Contaminación
MIP;

Monitoreo y uso

focalizado de plaguicidas;

Organización y cogestión;

Equipo de protección (para

tóxicos, térmica y ruido);

Programa técnico de

hidratación;

Ejercicio físico cotidiano;

Equipamiento ergonómico;

Programación detareas

acordecon condiciones

especiales de género

(ejemplo: embarazo)

Transporteseguro

Régimen de reposo diario y periódico;

Perfeccionamiento

y educación continuada

Identidad personal y

colectiva;

Conciencia ecológica

y social solidaria

Organización y

participación conciente

Manejo ecológico
- Deterioro mental y

fisiológico del trabajador/a

Alta emisión y empleo de

sust. decapacidad de daño:

Persistencia;

Bioacumulación;

Biomagnificación;

Potencialidad de

transporte

- Rutas de absorción

(ingestión, inhalación

dérmica)

- Formas/grados de acción

detóxicos:

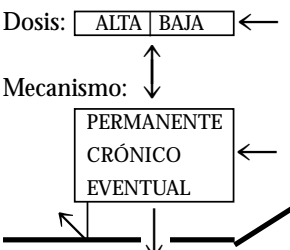

- Normas protectorasy

modalidades del código

deconducta:

Sistema de seguridad

y protección;

Nutrición adecuada;

Régimen de reposo y

jercicio físico protector

- Perfil psico-comunitario

y cultural protector 
Cuadro 2 (continuación)

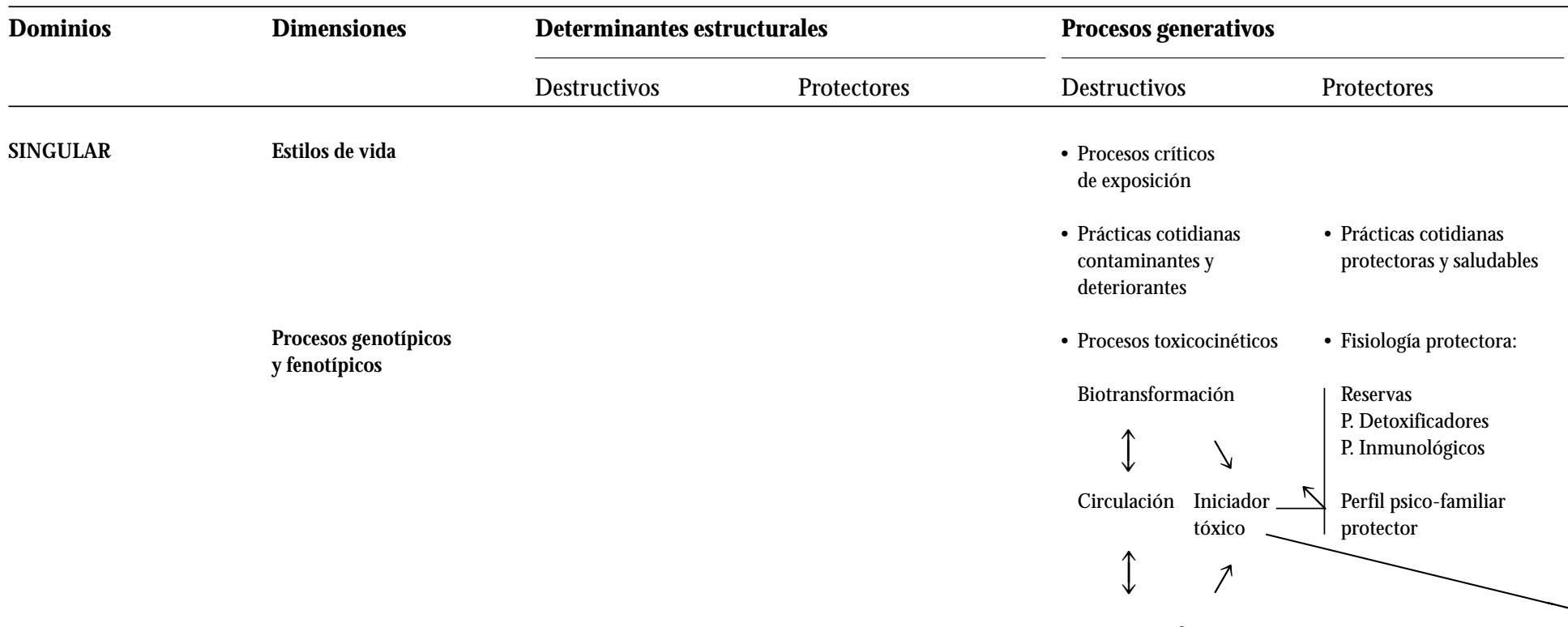

Fijación tisular

Procesosesp. desalud

(2)




\section{Referencias bibliográficas}

AyresJ 1997. Sobre o risco. Hucitec, São Paulo.

Betto (Frei) 2002. Economía y ciudadanía. Servicio Informativo deAlai-amlatina, São Paulo. Febrero 28.

Borón A 2002. Imperio \& imperialismo: una lectura crítica de $\mathrm{M}$ ichael $\mathrm{H}$ ardt y Antonio N egro. CLACSO, Buenos Aires.

Breilh J 1997. Vigilancia clásica o monitoreo estratégico en salud, pp. 203-221. En Nuevos conceptos y técnicas de investigación. (3era ed.). Ediciones del Centro de Estudios y Asesoría en Salud, Quito.

Breilh J 1999a. Derrota del conocimiento por la información. Ciência e Saúde Coletiva 5(1): 99-114.

Breilh J 1999b. Lineamientos para la integración de un sistema de información integrado y participativo. Documentos Técnicos del CEAS, Quito.

Breilh J 2002. De la gerencia del conocimiento a la planeación del saber. Ambato: Ponencia Presentada a Reunión de GTZ sobre "Gestión y Productos del Conocimiento", mayo

Breilh J 2003a. Epidemiología crítica: ciencia emancipadora einterculturalidad. Lugar Editorial, Buenos Aires.
Breilh J 2003b. Perspectivas políticas, sociales y éticas de la investigación en una era de barbarie. II Encuentro Internacional de Investigación en Enfermería, Águas de Lindóia (SP).

Corbière E 2002. El mito de la globalización capitalista: socialismo o barbarie. E-libro, net. Enero

Habermas J 1988. Teoría de la acción comunicativa. Taurus, $M$ adrid.

Hardt M \& Negri A 2000. Imperio. (trad. de Eduardo Sadier). Harvard, M assachusetts.

M észáros I 2001. Socialism or barbarism in "Imperialism and Empire" (John Bellamy Foster). M onthly Review 53(4):5-37.

Walsh C 200. ¿Qué conocimientos? Reflexiones sobre las políticas de conocimiento, el campo académico y el movimiento indígena ecuatoriano. Boletín del ICCI, № 25, abril.

Artigo apresentado em 5/10/2003

Aprovado em 30/102003

Versão final apresentada em 6/11/2003 